\title{
WHEN IS A MULTIPLICATIVE DERIVATION ADDITIVE?
}

\author{
MOHAMAD NAGY DAIF \\ Department of Mathematics \\ Faculty of Education \\ l'mm Al-Qura L'niversity \\ Taif, Saudi Arabia
}

(Received March 29, 1990 and in revised form December 19, 1990)

ABSTRACT. Our main objective in this note is to prove the following. Suppose $R$ is a ring having an idempotent element $e(e \neq 0, e \neq 1)$ which satisfies:

$\left(\mathrm{N}_{1}\right) \mathrm{xR}=0$ implies $\mathrm{x}=0$.

$\left(N_{2}\right)$ eRx $=0$ implies $x=0$ (and hence $R x=0$ implies $x=0$ ).

$\left(M_{3}\right)$ exeR $(1-e)=0$ implies exe $=0$.

If $d$ is any multiplicative derivation of $R$, then $d$ is additive.

KEY WORDS AND PHRASES. Ring, idempotent element, derivation, Peirce decomposition. 1980 AMS SUBJECT CLASSIFICATION CODES. 16A15, 16 A70.

1. INTRODUCTION.

In [1], Martindale has asked the following question : When is a multiplicative mapping additive? He answered his question for a multiplicative isomorphism of a ring $R$ under the existence of a family of idempotent elements in $R$ which satisfies some conditions.

Over the past few years, many results concerning derivations of rings have been obtained. In this note, we introduce the definition of a multiplicative derivation of a ring $R$ to be a mapping $d$ of $R$ into $R$ such that $d(a b)=d(a) b+a d(b)$, for $a 11 \mathrm{a}, \mathrm{b}$ in $\mathrm{R}$. As Martindale did, we raise the following question : When is a multiplicative derivation additive? Fortunately, we can give a full answer for this question using Martindale's conditions when assumed for a single fixed idempotent in $R$.

In the ring $R$, let $e$ be an idempotent element so that $e \neq 0$, $e \neq 1$ ( $R$ need not have an identity). As in [2], the two-sided Peirce decomposition of $R$ relative to the idempotent $e$ takes the form $R=e \operatorname{Re} \oplus e R(1-e) \oplus(1-e) \operatorname{Re} \oplus(1-e) R(1-e)$. We will forma$11 y$ set $e_{1}=e$ and $e_{2}=1-e$. So letting $R_{m n}=e_{m} R_{n} ; m, n=1,2$, we may write $R=R_{11} \biguplus$ $R_{12} \oplus R_{21} \oplus R_{22}$. Moreover, an element of the subring $R_{m n}$ will be denoted by $x_{m n}$.

From the definition of $d$ we note that $d(0)=d(00)=d(0) 0+0 d(0)=0$. Moreover, we have $d(e)=d\left(e^{2}\right)=d(e) e+e d(e)$. So we can express $d(e)$ as $a_{11}+a_{12}+a_{21}+a_{22}$ and use the value of $d(e)$ to get that $a_{11}=a_{22}$, that is, $a_{11}=0=a_{22}$. Consequently, we have $d(e)=a_{12}+a_{21}$.

Now let $f$ be the inner derivation of $R$ determined by the element $a_{12}-a_{21}$, that is $f(x)=\left[x, a_{12}-a_{21}\right]$ for all $x$ in $R$. Therefore, $f(e)=\left[e, a_{12}-a_{21}\right]=a_{12}+a_{21}$. 
In the sequel, and without loss of generality, we can replace the multiplicative derivation $d$ by the multiplicative derivation $d-f$, which we denote by $D$, that is, $D=d-f$. This yields $D(e)=0$. This simplification is of great importance, for, as we will see, the subrings $R_{m n}$ become invariant under the multiplicative derivation D.

2. A KEY LEMMA.

LEMMA 1. $D\left(R_{m n}\right) \subset_{m n}, m, n=1,2$.

PROOF. Let $x_{11}$ be an arbitrary element of $R_{11}$. Then $D\left(x_{11}\right)=D\left(e x_{11} e\right)=e D\left(x_{11}\right) e$ which is an element of $R_{11}$. For an element $x_{12}$ in $R_{12}$, we have $D\left(x_{12}\right)=D\left(e x_{12}\right)=$ $e D\left(x_{12}\right)=b_{11}+b_{12}$. But $0=D(0)=D\left(x_{12} e\right)=D\left(x_{12}\right) e=b_{11}$, hence $D\left(x_{12}\right)=b_{12}$ which belongs to $R_{12}$. In a similar fashion, for an element $x_{21}$ in $R_{21}$, we have $D\left(x_{21}\right)$ belongs to $R_{21}$. Now take an element $x_{22}$ in $R_{22}$. Write $D\left(x_{22}\right)=c_{11}+c_{12}+c_{21}+c_{22}$. So, $0=D\left(e_{22}\right)=e D\left(x_{22}\right)=c_{11}+c_{12}$, whence $c_{11}=c_{12}=0$. Likewise $c_{21}=0$, and thus $D\left(x_{22}\right)=c_{22}$ which is an element of $R_{22}$. This proves the lemma.

3. CONDITIONS OF MARTINDALE.

In his note [1], Martindale has given the following conditions which are imposed on a ring $R$ having a family of idempotent elements $\left\{e_{i}: i \in I\right\}$.

(1) $x R=0$ implies $x=0$.

(2) If $e_{i} R x=0$ for each $i$ in $I$, then $x=0$ (and hence $R x=0$ implies $x=0$ ).

(3) For each $i$ in $I, e_{i} x e_{i} R\left(1-e_{i}\right)=0$ implies $e_{i} x e_{i}=0$.

In our note, we find it appropriate to simply dispense with conditions (1), (2)

and (3) altogether and instead substitute the following conditions :

$\left(M_{1}\right) \times R=0$ implies $x=0$.

$\left(M_{2}\right)$ eRx $=0$ implies $x=0$ (and hence $R x=0$ implies $x=0$ ).

$\left(\mathrm{N}_{3}\right) \operatorname{exeR}(1-\mathrm{e})=0$ implies exe $=0$.

4. AUXILIARY LEMMIAS.

LEMMA 2. For any $x_{m m}$ in $R_{m m}$ and any $x_{p q}$ in $R_{p q}$ with $p \neq q$, we have

$$
\mathrm{D}\left(\mathrm{x}_{\mathrm{mm}}+\mathrm{x}_{\mathrm{pq}}\right)=\mathrm{D}\left(\mathrm{x}_{\mathrm{mm}}\right)+\mathrm{D}\left(\mathrm{x}_{\mathrm{pq}}\right) \text {. }
$$

PROOF. Assume $\mathrm{m}=\mathrm{p}=1$ and $\mathrm{q}=2$.

Consider the sum $D\left(x_{11}\right)+D\left(x_{12}\right)$. Let $t_{1 n}$ be an element of $R_{1 n}$. Using Lemm 1 , we have $\left[D\left(x_{11}\right)+D\left(x_{12}\right)\right] t_{1 n}=D\left(x_{11}\right) t_{1 n}=D\left(x_{11} t_{1 n}\right)-x_{11} D\left(t_{1 n}\right)=D\left[\left(x_{11}+x_{12}\right) t_{1 n}\right]-$ $x_{11} D\left(t_{1 n}\right)=D\left(x_{11}+x_{12}\right) t_{1 n}+\left(x_{11}+x_{12}\right) D\left(t_{1 n}\right)-x_{11} D\left(t_{1 n}\right)=D\left(x_{11}+x_{12}\right) t_{1 n}$. Thus, $\left[D\left(x_{11}\right)+D\left(x_{12}\right)-D\left(x_{11}+x_{12}\right)\right] t_{1 n}=0$.

In the same fashion, for any $t_{2 n}$ in $R_{2 n}$, we can get the following

$$
\left[D\left(x_{11}\right)+D\left(x_{12}\right)-D\left(x_{11}+x_{12}\right)\right] t_{2 n}=0 \text {. }
$$

Combining these results, we have $\left[D\left(x_{11}\right)+D\left(x_{12}\right)-D\left(x_{11}+x_{12}\right)\right] R=0$. By condition $\left(M_{1}\right)$, we obtain

$$
D\left(x_{11}+x_{12}\right)=D\left(x_{11}\right)+D\left(x_{12}\right) .
$$

In view of the symmetry resulting from condition $\left(M_{1}\right)$ and the implication of condition $\left(\mathrm{M}_{2}\right)$, we can find that the other three cases are easily shown in a similar fashion.

LEMMA 3. $D$ is additive on $R_{12}$.

PROOF. Let $x_{12}$ and $y_{12}$ be two elements in the subring $R_{12}$, and consider the sum 
$D\left(x_{12}\right)+D\left(y_{12}\right)$.

(A) For an element $t_{1 n}$ in $R_{1 n}$, we have $\left[D\left(x_{12}\right)+D\left(y_{12}\right)\right] t_{1 n}=D\left(x_{12}+y_{12}\right) t_{1 n}$, since each side is zero by Lemma 1 , so

$$
\left[D\left(x_{12}\right)+D\left(y_{12}\right)-D\left(x_{12}+y_{12}\right)\right] t_{1 n}=0 .
$$

(B) Consider an element $t_{2 n}$ in $R_{2 n}$. We have $\left(x_{12}+y_{12}\right) t_{2 n}=\left(e+x_{12}\right)\left(t_{2 n}+\right.$ $\left.y_{12} t_{2 n}\right)$. Thus, $D\left[\left(x_{12}+y_{12}\right) t_{2 n}\right]=D\left(e+x_{12}\right)\left(t_{2 n}+y_{12} t_{2 n}\right)+\left(e+x_{12}\right) D\left(t_{2 n}+y_{12} t_{2 n}\right)$ $=\left(D(e)+D\left(x_{12}\right)\right)\left(t_{2 n}+y_{12} t_{2 n}\right)+\left(e+x_{12}\right)\left(D\left(t_{2 n}\right)+D\left(y_{12} t_{2 n}\right)\right)=D\left(x_{12}\right) t_{2 n}+x_{12} D\left(t_{2 n}\right)$ $+D\left(y_{12} t_{2 n}\right)$, by Lemmas 1 and 2. Thus, $D\left(\left(x_{12}+y_{12}\right) t_{2 n}\right)=D\left(x_{12} t_{2 n}\right)+D\left(y_{12} t_{2 n}\right)$. But $\left(D\left(x_{12}\right)+D\left(y_{12}\right)\right) t_{2 n}=D\left(x_{12}\right) t_{2 n}+D\left(y_{12}\right) t_{2 n}=D\left(x_{12} t_{2 n}\right)+D\left(y_{12} t_{2 n}\right)-\left(x_{12}+y_{12}\right) D\left(t_{2 n}\right)=$ $D\left(\left(x_{12}+y_{12}\right) t_{2 n}\right)-\left(x_{12}+y_{12}\right) D\left(t_{2 n}\right)=D\left(x_{12}+y_{12}\right) t_{2 n}$. Hence,

$$
\left[D\left(x_{12}\right)+D\left(y_{12}\right)-D\left(x_{12}+y_{12}\right)\right] t_{2 n}=0 \text {. }
$$

Consequently, from $(A)$ and $(B)$ we have

By condition $\left(M_{1}\right)$, we have

$$
\left[D\left(x_{12}\right)+D\left(y_{12}\right)-D\left(x_{12}+y_{12}\right)\right] R=0 .
$$

LEMMA 4. $D$ is additive on $R_{11}$.

$$
D\left(x_{12}+y_{12}\right)=D\left(x_{12}\right)+D\left(y_{12}\right) .
$$

PROOF. Let $x_{11}$ and $y_{11}$ be arbitrary elements in $R_{11}$. For an element $t_{12}$ in $R_{12}$, we have $\left(D\left(x_{11}\right)+D\left(y_{11}\right)\right) t_{12}=D\left(x_{11}\right) t_{12}+D\left(y_{11}\right) t_{12}=D\left(x_{11} t_{12}\right)+D\left(y_{11} t_{12}\right)-\left(x_{11}+\right.$ $\left.y_{11}\right) D\left(t_{12}\right)$. But $x_{11} t_{12}$ and $y_{11} t_{12}$ are in $R_{12}$, and $D$ is additive on $R_{12}$ by Lemma 3 , hence $\left(D\left(x_{11}\right)+D\left(y_{11}\right)\right) t_{12}=D\left(x_{11} t_{12}+y_{11} t_{12}\right)-\left(x_{11}+y_{11}\right) D\left(t_{12}\right)=D\left(\left(x_{11}+y_{11}\right) t_{12}\right)$ $-\left(x_{11}+y_{11}\right) D\left(t_{12}\right)=D\left(x_{11}+y_{11}\right) t_{12}$. thus we have

Therefore,

$$
\left[D\left(x_{11}\right)+D\left(y_{11}\right)-D\left(x_{11}+y_{11}\right)\right] t_{12}=0 .
$$

$$
\left[D\left(x_{11}\right)+D\left(y_{11}\right)-D\left(x_{11}+y_{11}\right)\right] R_{12}=0 .
$$

From Lemma $1, D\left(x_{11}\right)+D\left(y_{11}\right)-D\left(x_{11}+y_{11}\right)$ is an element in $R_{11}$, hence the above result with condition $\left(M_{3}\right)$ give

$$
D\left(x_{11}+y_{11}\right)=D\left(x_{11}\right)+D\left(y_{11}\right) \text {. }
$$

LEMMA 5. $D$ is additive on $R_{11}+R_{12}=e R$.

PROOF. Consider the arbitrary elements $x_{11}, y_{11}$ in $R_{11}$ and $x_{12}, y_{12}$ in $R_{12}$. So, Lemmas $2,3,4$ give $D\left(\left(x_{11}+x_{12}\right)+\left(y_{11}+y_{12}\right)\right)=D\left(\left(x_{11}+y_{11}\right)+\left(x_{12}+y_{12}\right)\right)=D\left(x_{11}+\right.$ $\left.y_{11}\right)+D\left(x_{12}+y_{12}\right)=D\left(x_{11}\right)+D\left(y_{11}\right)+D\left(x_{12}\right)+D\left(y_{12}\right)=\left(D\left(x_{11}\right)+D\left(x_{12}\right)\right)+\left(D\left(y_{11}\right)\right.$ $\left.+D\left(y_{12}\right)\right)=D\left(x_{11}+x_{12}\right)+D\left(y_{11}+y_{12}\right)$. Thus $D$ is additive on $R_{11}+R_{12}$. This proves the desired result.

5. MAIN THEOREM.

THEOREM. Let $R$ be a ring containing an idempotent $e$ which satisfies conditions $\left(M_{1}\right),\left(M_{2}\right)$ and $\left(M_{3}\right)$. If $d$ is any multiplicative derivation of $R$, then $d$ is additive.

PROOF. As we mentioned before, and without loss of generality, we can replace $d$ by $D$. Let $x$ and $y$ be any elements of $R$. Consider $D(x)+D(y)$. Take an element $t$ in eR $=R_{11}+R_{12}$. Thus, $t x$ and $t y$ are elements of $e R$. According to Lemma 5 , we can obtain $t(D(x)+D(y))=t D(x)+t D(y)=D(t x)+D(t y)-D(t)(x+y)=D(t x+t y)-D(t(x+y))$ 
$+t D(x+y)$. Thus, $t(D(x)+D(y))=t D(x+y)$. Since $t$ is arbitrary in eR, we obtain $e R(D(x)+D(y)-D(x+y))=0$. By condition $\left(M_{2}\right)$, we get

$$
D(x+y)=D(x)+D(y) \text {. }
$$

which shows that the multiplicative derivation $D$ is additive.

ACKNOWLEDGEMENT. The author is indebted to the referee for his helpful suggestions and valuable comments which helped in appearing the paper in its present shape.

\section{REFERENCES}

1. MARTINDALE III, h.S. When are Multiplicative Mappings Additive ?, Proc. Amer. Math. Soc. 21 (1969), 695-698.

2. JACOBSON, N. Structure of Rings, Amer. Math. Soc. Collog. Publ. 37 (1964). 


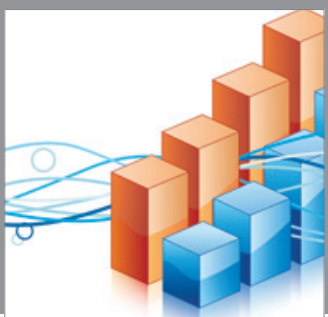

Advances in

Operations Research

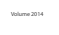

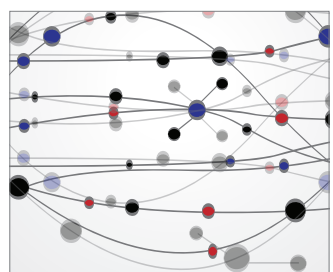

\section{The Scientific} World Journal
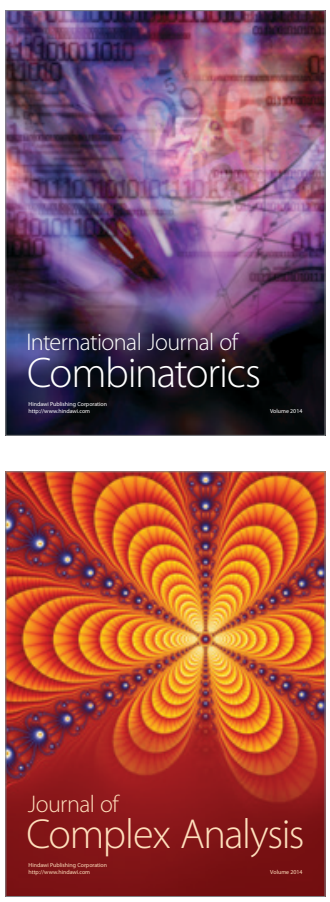

International Journal of

Mathematics and

Mathematical

Sciences
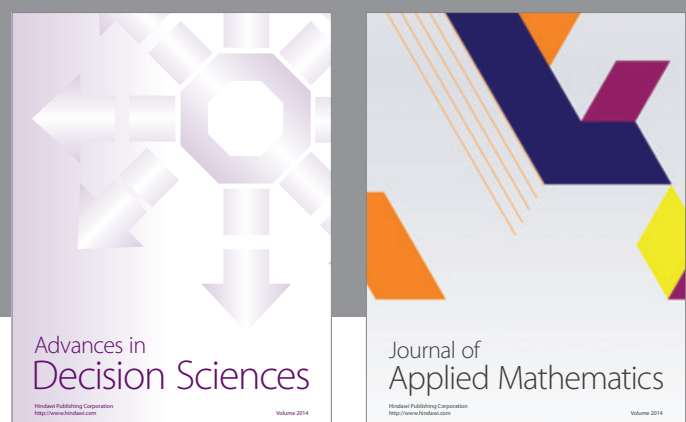

Journal of

Applied Mathematics
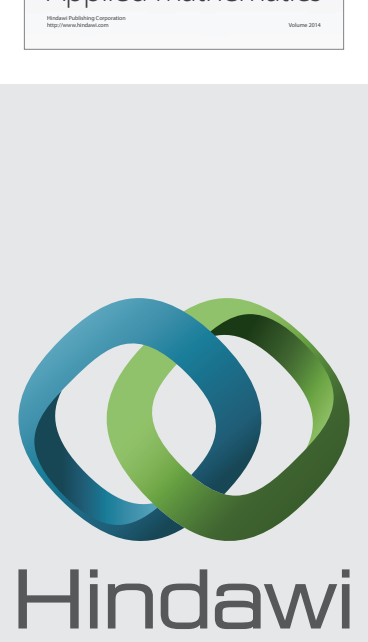

Submit your manuscripts at http://www.hindawi.com
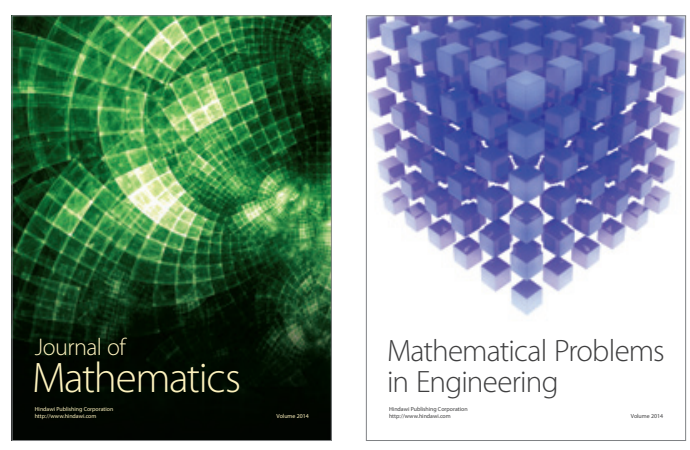

Mathematical Problems in Engineering
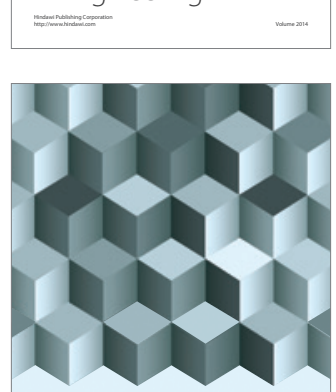

Journal of

Function Spaces
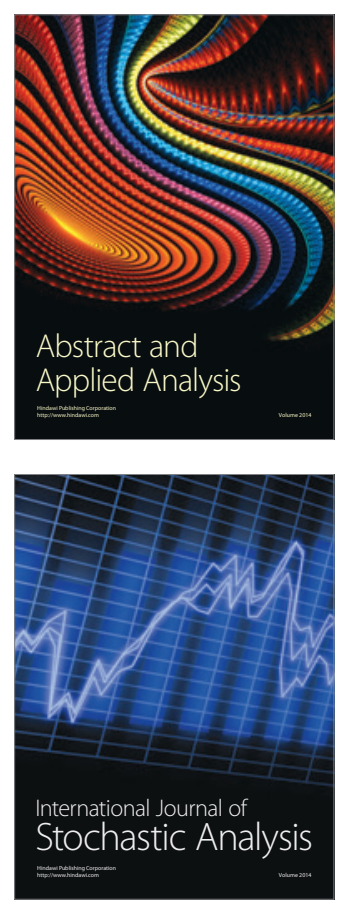

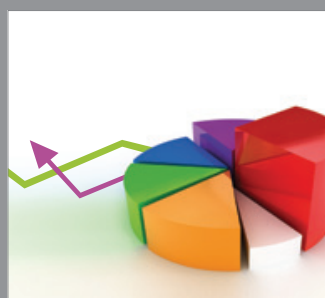

ournal of

Probability and Statistics

Promensencen
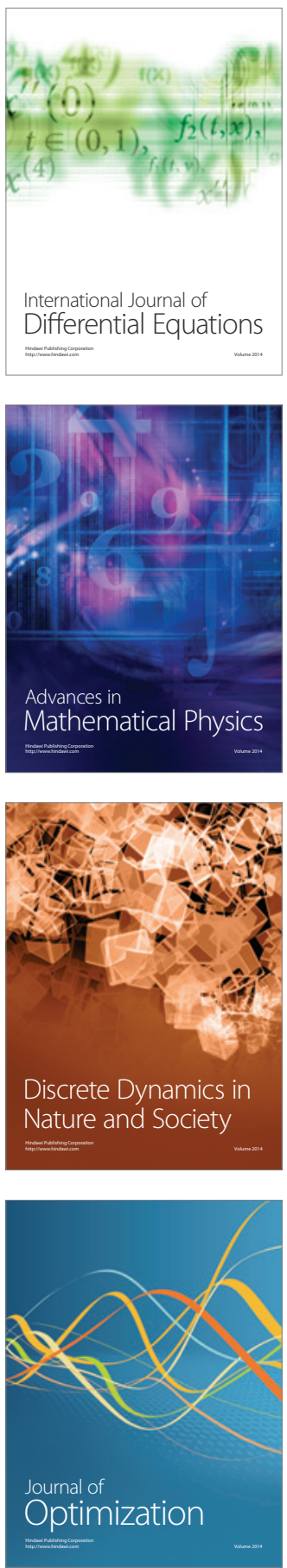